\title{
IMPACT OF TRAFFIC NOISE ON TIBETAN ANTELOPES: A PRELIMINARY EXPERIMENT ON THE QINGHAI-TIBET HIGHWAY IN CHINA
}

\author{
RU, H. ${ }^{1}-$ XU, J. ${ }^{{ }^{*}}-\mathrm{LI}, \mathrm{M} .{ }^{1}-\mathrm{DUAN}, \mathrm{Z}{ }^{1}-\mathrm{LI}, \mathrm{Z}^{2}$ \\ ${ }^{1}$ College of highway, Chang'an University \\ $X i$ 'an 710064, China \\ (e-mail:435516439@qq.com; phone: +86-15686209885) \\ ${ }^{2}$ Shanxi Academy of Urban \& Rural Planning and Design \\ Taiyuan 030001, China \\ *Corresponding author \\ e-mail:2016021066@chd.edu.cn; phone: +86-18700948223 \\ (Received $4^{\text {th }}$ Feb 2018; Accepted $26^{\text {th }}$ Apr 2018)
}

\begin{abstract}
The traffic noise on a highway with a non-negligible negative impact on the behavior of wild animals has a great ecological impact on wildlife near the road area. To analyze the impact of traffic noise on Tibetan antelopes, field experiments were carried out to measure the traffic noise intensity and observe the behavior of the Tibetan antelope within $500 \mathrm{~m}$ of the Qinghai-Tibet Highway from Kunlun Mountain Pass to Wudaoliang on the Qinghai-Tibet Plateau, China. A relationship between different levels of traffic noise intensity and the behavior of Tibetan antelope was established by data analysis. Traffic levels were found to have the most significant effect on Tibetan antelope behavior within $150 \mathrm{~m}$ of the highway. The changes in the Tibetan antelope behavior before and after changes in noise mainly manifested as decreased foraging and increased movement. The results of this study can guide road construction and engineering measures to protect wildlife on the Qinghai-Tibet Plateau and other areas with high altitude.
\end{abstract}

Keywords: road ecology, traffic noise, noise pollution, Tibetan antelope behavior, high altitude area

\section{Introduction}

The traffic noise from a highway has a large ecological impact on wild animals near road area (Forman et al., 1998). Growing evidence has shown that traffic noise has a non-negligible negative impact on wild animals' behavior. The impact mainly manifests as avoidance of the road vicinity (Zande et al., 1980). Traffic noise may also obstruct acoustic communication among wild animals that depend on it, including frogs, birds, and mammals (Parris et al., 2009). The negative impact on wild animal fitness at the individual level may have severe population-level consequences because most of such wildlife are social animals. Thus, wildlife populations living near road areas exposed to traffic noise have a high potential for reduced reproductive success, which may ultimately break the local ecological balance.

The impact of roads on wildlife has drawn wide attention in recent years. A study in Canada measured the extent and type of relationship underlying the road-effect zones of a motorway with a high proportion of heavy-truck traffic for anuran species richness (Eigenbrod et al., 2009). Field experiments were performed to study the road-effect zones of ungulate wild animals in the USA (Gagnon et al., 2007). To analyze the effect of vehicular traffic on wild animals, a study in India estimated the road kills on state highways (Samson et al., 2016). New technologies have also been used in various studies. To analyze the effects of the highway transportation corridors on wildlife, a 
study used a geographic information system (GIS) to identify landscape attributes associated with species movement in Banff National Parka, Canada (Alexander et al., 2000). To locate accident points and acquire spatial statistics for analysis, a study utilized a GIS to research methods for mitigating wildlife-vehicle collisions in Spain (Diaz-Varela et al., 2011).

Most existing research on the impact of traffic noise on wildlife has mainly focused on birds. Field experiments have indicated a range of behavioral responses to urban noise in North America and Seville, Spain (Wood et al., 2006; Slabbekoorn et al., 2008). Traffic noise has also been shown to affect wildlife migration and reproduction. Parris and Schneider investigated the impact of traffic noise and traffic volume on two bird species in Australia, and found that traffic noise may reduce breeding success in noisy roadside habitats (Parris et al., 2009). The negative impact of traffic noise on avian reproductive success was analyzed in Netherlands (Halfwerk et al., 2011). However, there have been only a few studies that focused on the impact of traffic noise on mammals around the world.

The impact of traffic noise on wildlife has been extensively studied for various geographic topographies around the world. For instance, road kills were analyzed for state highways on Sigur Plateau, India (Gagnon et al., 2007). The effects of traffic noise on bird populations were studied for mountain forests in Idaho, USA (Mcclure et al., 2013). In China, the Qinghai-Tibet Plateau is a unique region with a high altitude and fragile ecological environment. Many wild animals, especially Tibetan antelope, are distributed around the Qinghai-Tibet Highway. The impact of roads on Tibetan antelope has received attention in recent years. Research has mainly focused on the road-effect zone (i.e., extent of significant ecologic effects from the edge of a road) and the impact on migration and protection measures of Tibetan antelopes (Lian et al., 2012; Lin et al., 2007). Traffic noise is known to have an impact on wildlife behavior. However, there has been no research on the impact of traffic noise on Tibetan antelopes and the extent of this impact.

In this study, the impact of different levels of traffic noise intensity on the population behavior of Tibetan antelopes was examined. Field experiments were performed to measure the traffic noise intensity and observe the behavior of the Tibetan antelope within $500 \mathrm{~m}$ of the Qinghai-Tibet Highway from Kunlun Mountain Pass to Wudaoliang on the Qinghai-Tibet Plateau, China. The relationship between different levels of traffic noise intensity and the behavior of Tibetan antelope was analyzed based on the traffic noise intensity data and corresponding proportions of different kinds of Tibetan antelope behaviors at different distances from the highway.

The remainder of the paper is organized as follows. First, the field experiments and data collection process are described in detail. Then the experimental results are analyzed, followed by a discussion and insights for future research.

\section{Materials and Methods}

\section{Research Area}

The research area was located at the junction connecting Hoh Xil National Nature Reserve and Sanjiang Yuan Nature Reserve: the crossing of Qinghai-Tibet Highway from Kunlun Mountain Pass (K2900, 4767 m ASL) to Wudaoliang (K3005, $4800 \mathrm{~m}$ ASL) (Fig. 1). The geographic structures of the research area include a plateau mountain and plateau grassland for a high-altitude environment. This is a favorable 
habitat for Tibetan antelope, especially from Chumar River to Wudaoliang. The migration season of Tibetan antelope is usually from June to August, so June was selected for surveying. Qinghai-Tibet Highway traverses the annual migration path of Tibetan antelopes. The geographic location of Qinghai-Tibet highway ensures that the main source of noise pollution is traffic. Thus, this $105 \mathrm{~km}$ long stretch of the highway from Kunlun Mountain Pass to Wudaoliang was selected for surveying.

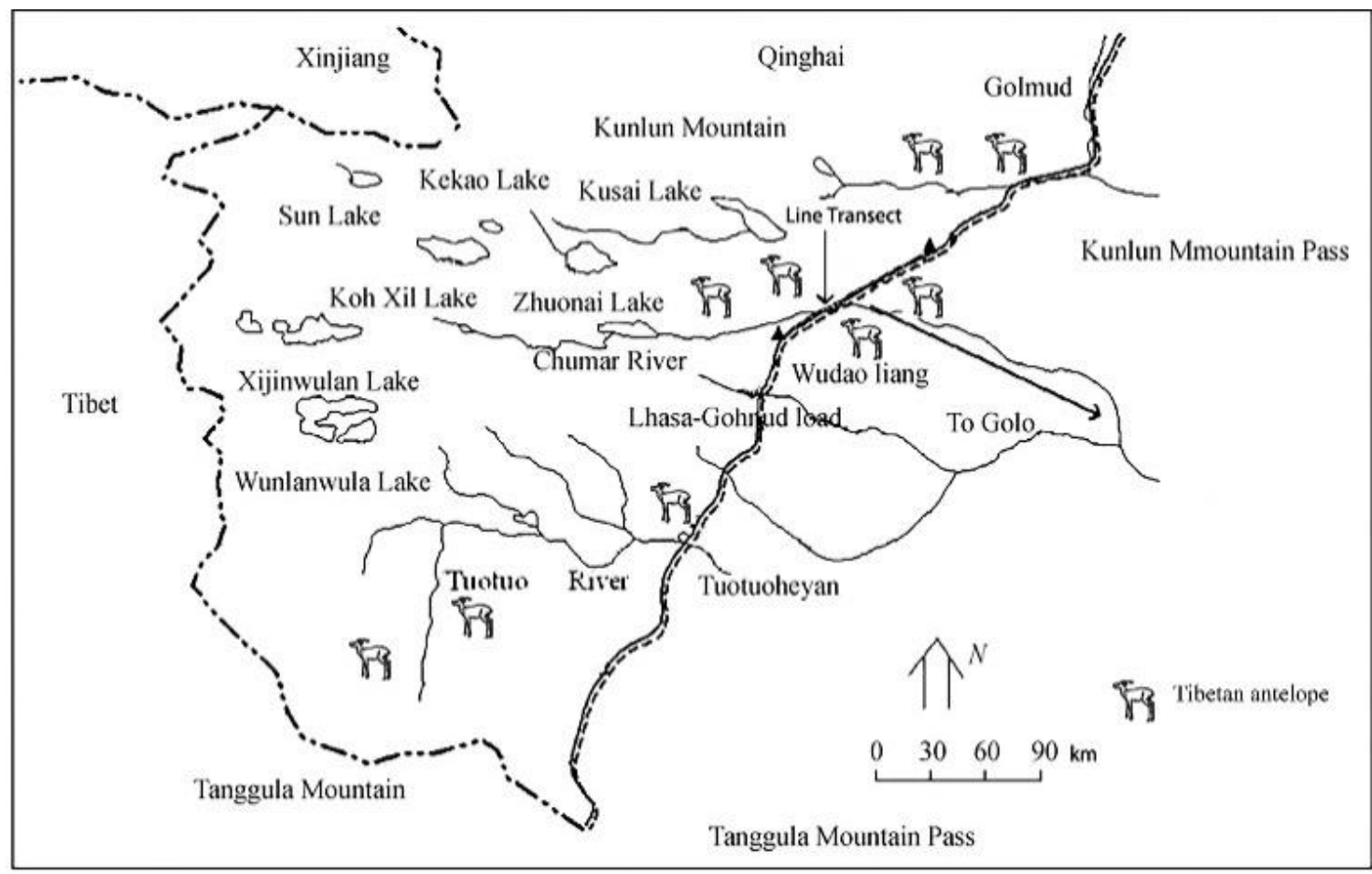

Figure 1. Survey area

\section{Field Experiment}

To analyze the impact of traffic noise on Tibetan antelope behavior, the influence of different levels of traffic noise intensity on the behavior of Tibetan antelope was measured. There are three key nodes in the research area along the migration path of Tibetan antelope: K2904 (i.e., the wildlife channel between the Kunlun Mountains and Qingshui River), K2965 (i.e., Chubei Channel), and K2998 (i.e., the channel between Chumar River and Hoh Xil). To determine the experiment site, these three nodes were surveyed three full daytime in June $1^{\text {st }}$ and $3^{\text {rd }}, 2016$. The results showed that a large amount of Tibetan antelope was distributed on the east side of the highway at K2998 (Fig. 2). For further research, the hourly traffic volume at K2998 was collected by using the AXLELIGHT Roadside Laser Vehicle Classification System in daytime June $4^{\text {th }}$ to $6^{\text {th }}, 2016$. The daily difference in traffic volume at K2998 was collected, and the results showed slight differences in the daily traffic volume. The traffic volume was greatest from 8:00 am to 10:00 am (126-138 pcu/h) and corresponds to the most unfavorable condition for Tibetan antelope. Therefore, the east side of Qinghai-Tibet Highway (K2998) from 8:00 am to 10:00 am was set as the experimental site for determining the impact of traffic noise on the behavior of Tibetan antelope. 


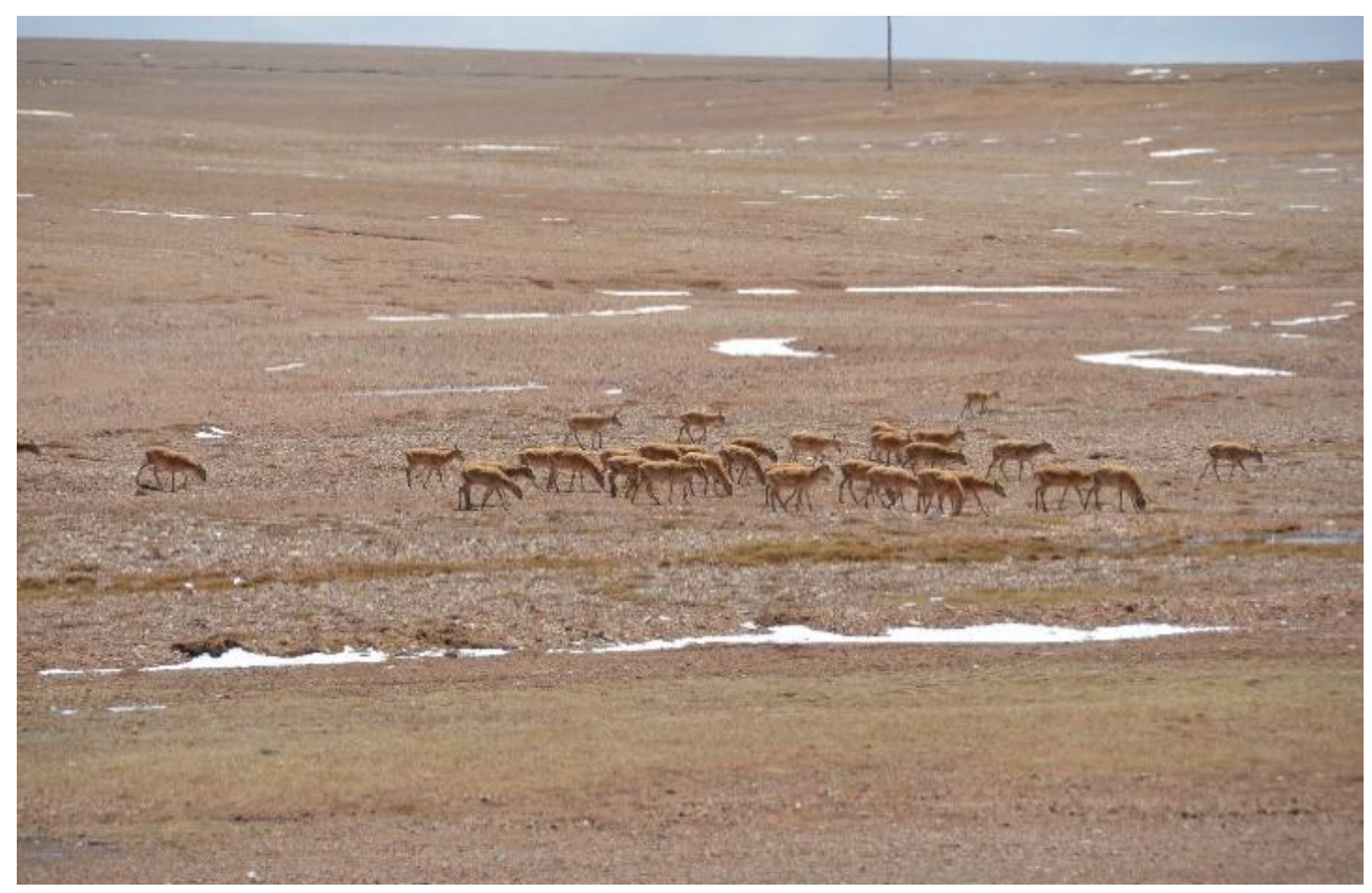

Figure 2. Tibetan antelope population at K2998

\section{Noise Measures}

To avoid adverse factors that may affect the accuracy of the experimental results, such as visual or aural interference for Tibetan antelope during the noise intensity measurement, the traffic noise intensity was measured before the observation of Tibetan antelope behavior. To avoid visual interference, observers did not appear in front of the Tibetan antelope but stayed in the test vehicle. With the aim of keep consistency in weather for observation, four sunny days without windy were selected to measure noise during the date from Jun $4^{\text {th }}$ to Jun $11^{\text {st }}, 2016$, here it is worth to mention again that the observation time is identical four all the four discrete days for noise measures, from 8:00 am to 10:00 am.

The distance from the east roadside was demarcated along the highway by using a laser range finder. Within $500 \mathrm{~m}$ of the roadside, signs were marked every $50 \mathrm{~m}$ to acquire the approximate behavior of Tibetan antelope at different distances. The average noise intensity was measured with a noise meter having a resolution of $10 \mathrm{~m}$. The average noise intensity at a certain distance during a certain time period was calculated as follows:

$$
X_{j}=\sum \bar{x}_{i j}
$$

where, $X_{j}$ is the level of average noise intensity at distance $j$ during a certain time period and $\bar{x}_{i j}$ is the average noise intensity at distance $j$ and time $i$ during a certain time period. When a vehicle passed through the vertical intersection (i.e., shortest distance) between the experiment site and highway, the level of instantaneous noise was 
measured immediately, and the maximum value was selected as the maximum noise level during a certain time period.

\section{Behavioral Observations}

There are significant differences in the behavioral characteristics of Tibetan antelope depending on the research objective. Such behaviors can be classified as follows: foraging, vigilance, movement, rest, and others. Table 1 presents the main behavioral variables.

Table 1. Definitions of the behavioral variables for Tibetan antelope

\begin{tabular}{|c|c|}
\hline Behavior & Definitions of Behavioral Variables \\
\hline Foraging & Standing or walking with the head below the horizontal line of the shoulder \\
\hline Vigilance & Standing and watching a certain direction \\
\hline Movement & $\begin{array}{c}\text { Displacement of a single individual with the head parallel or above the horizontal line of } \\
\text { the shoulder }\end{array}$ \\
\hline Rest & Lying down on the ground \\
\hline Others & Playing, breastfeeding, defecation, etc. \\
\hline
\end{tabular}

Field experiments for seven days were carried out to observe the behavior of the Tibetan antelope during the date from $12^{\text {nd }}$ to $19^{\text {th }}$ June, 2016. Based on the statistical data, the average driving speed on this road section was around $50 \mathrm{~km} / \mathrm{h}$. Thus, the test vehicle was driven back and forth in the experiment site at a cruise speed of around 50 $\mathrm{km} / \mathrm{h}$. Meanwhile, three observers sitting in the test vehicle observed the proportions of different behaviors exhibited by the antelope being passed by at different distances within $500 \mathrm{~m}$ of the east roadside with the help of a high-powered telescope.

\section{Results}

\section{Level of Noise Changes at Different Distances from the Highway}

In this study, 500 datasets were collected regarding the level of average noise intensity and instantaneous noise intensity at different distances within $500 \mathrm{~m}$ along the east roadside. The levels of the average noise intensity and maximum noise intensity were calculated at different distances away from the roadside from 8:00 am to 10:00 am. The noise level intensity was associated with different distances from the highway, as shown in Figure 3. The horizontal axis represents the distance from the highway, and the vertical axis represents the noise in decibels.

The levels of average noise and maximum noise within $500 \mathrm{~m}$ of the highway differed by 3.58-21.15 dB. The difference between them gradually decreased with increasing distance from the highway and then tended to a stable value. However, the two curves representing the levels of average noise and maximum noise showed a generally consistent trend (i.e., nonlinear negative correlation): the noise level decreased with increasing distance from the highway. The noise level decreased sharply with increasing distance up to $150 \mathrm{~m}$ from the highway, but the two curves declined gently beyond this range. 


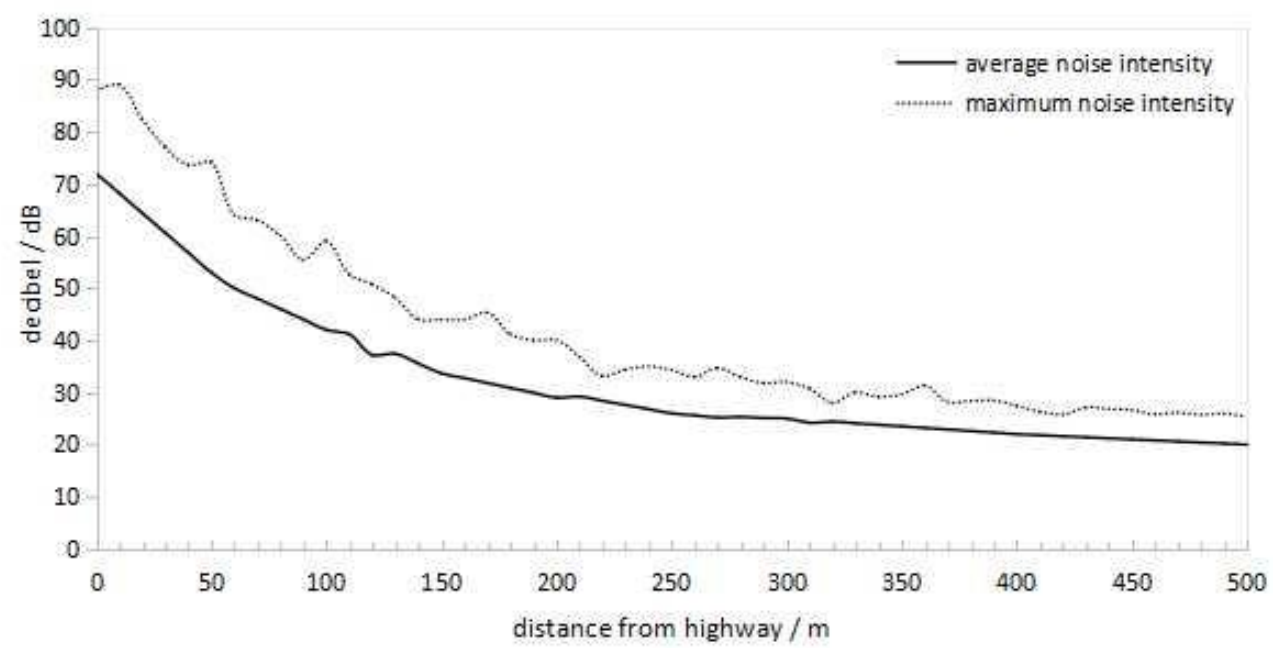

Figure 3. Change in noise level with distance

SPSS statistics 17.0 was used for statistical analysis to validate the data. Based on the normal distribution test method and the experimental data, two histograms were obtained, as shown in Figure 4. The horizontal axis represents the average noise and instantaneous noise in decibels, and the vertical axis represents the frequency that each decibel value occurred. The absolute values of the skewness and kurtosis were all less than 1. Therefore, the frequencies of the average and instantaneous noise intensity obeyed an approximately normal distribution.
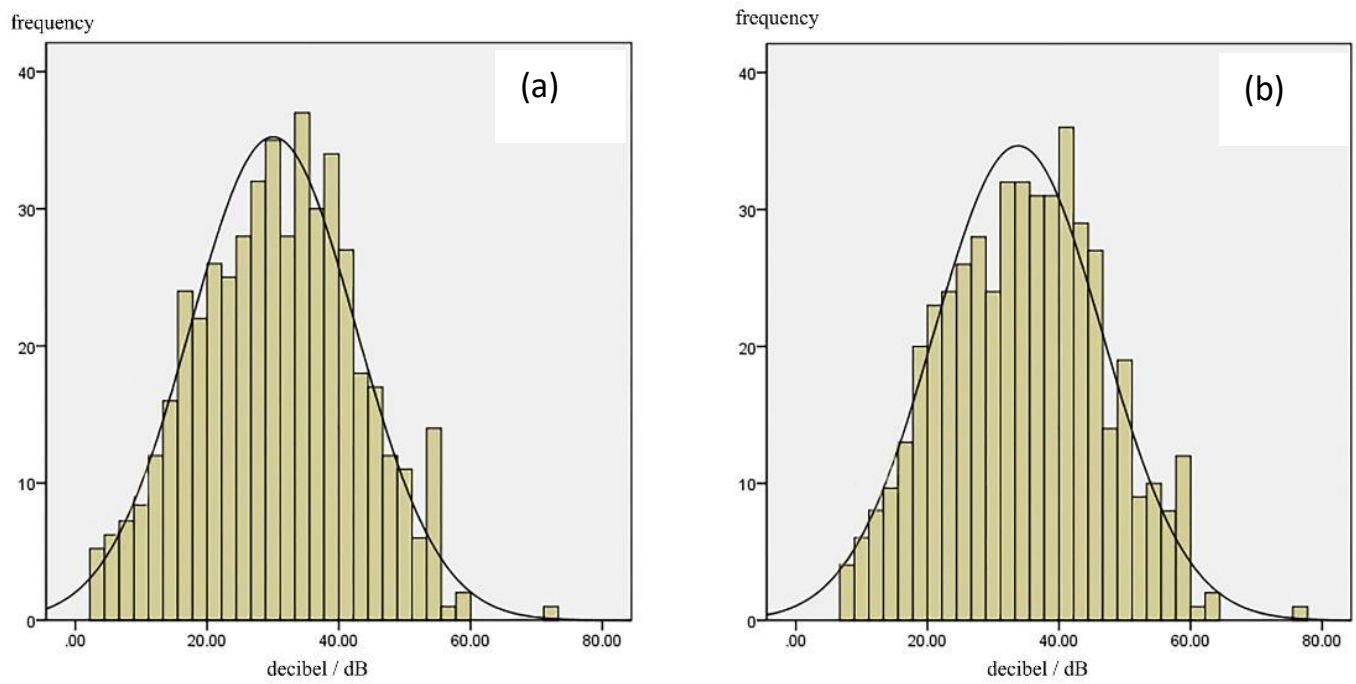

Figure 4. Distribution of noise intensity data: (a) average and (b) instantaneous noise intensities

\section{Changes in Tibetan Antelope Behavior with Different Levels of Traffic Noise}

In this study, 1033 Tibetan antelopes were observed, and their behaviors such as foraging, vigilance, movement, rest, and others were recorded. To analyze the association between the Tibetan antelope behavior and noise level, the proportions of 
different behaviors affected by the different noise levels were plotted as shown in Figure 5. The horizontal axis represents the percentage of each behavior, and the vertical axis represents the distance from the highway.

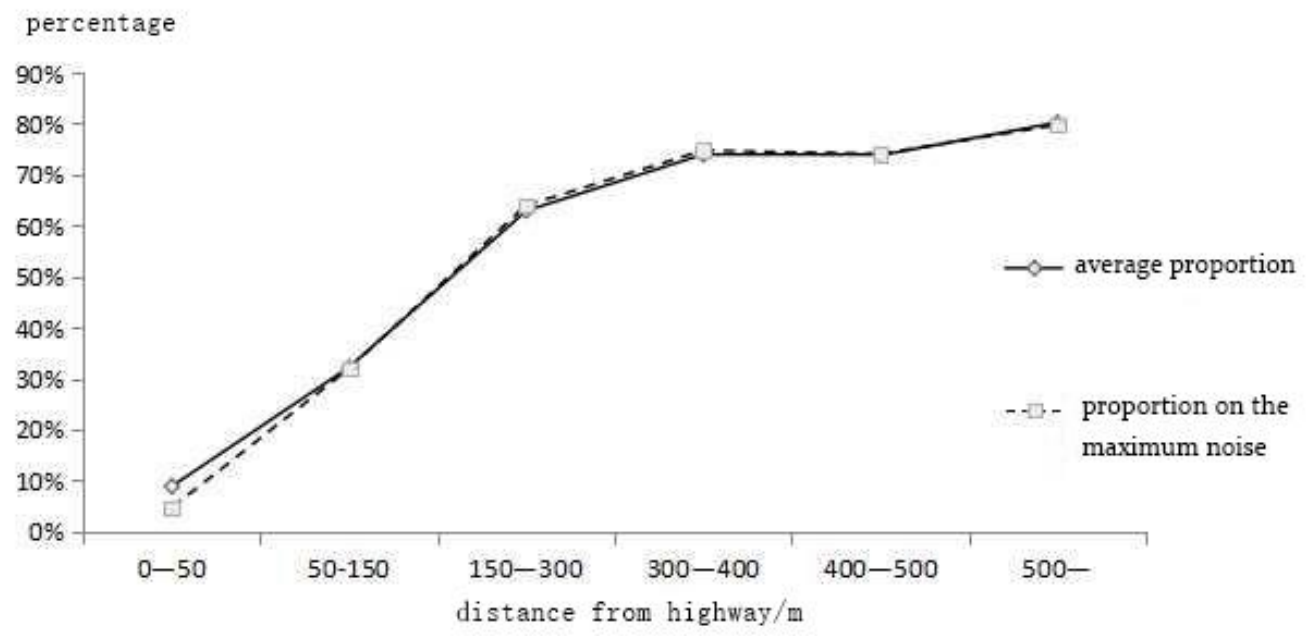

(a) Foraging

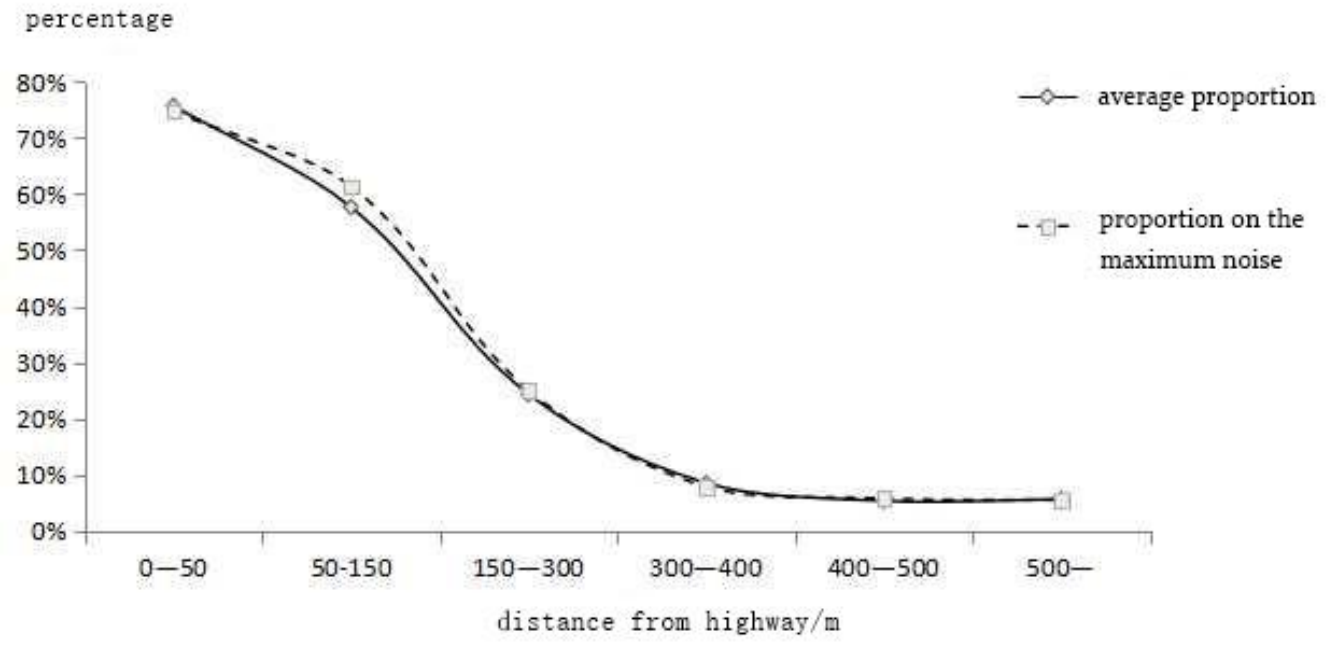

(b) Vigilance

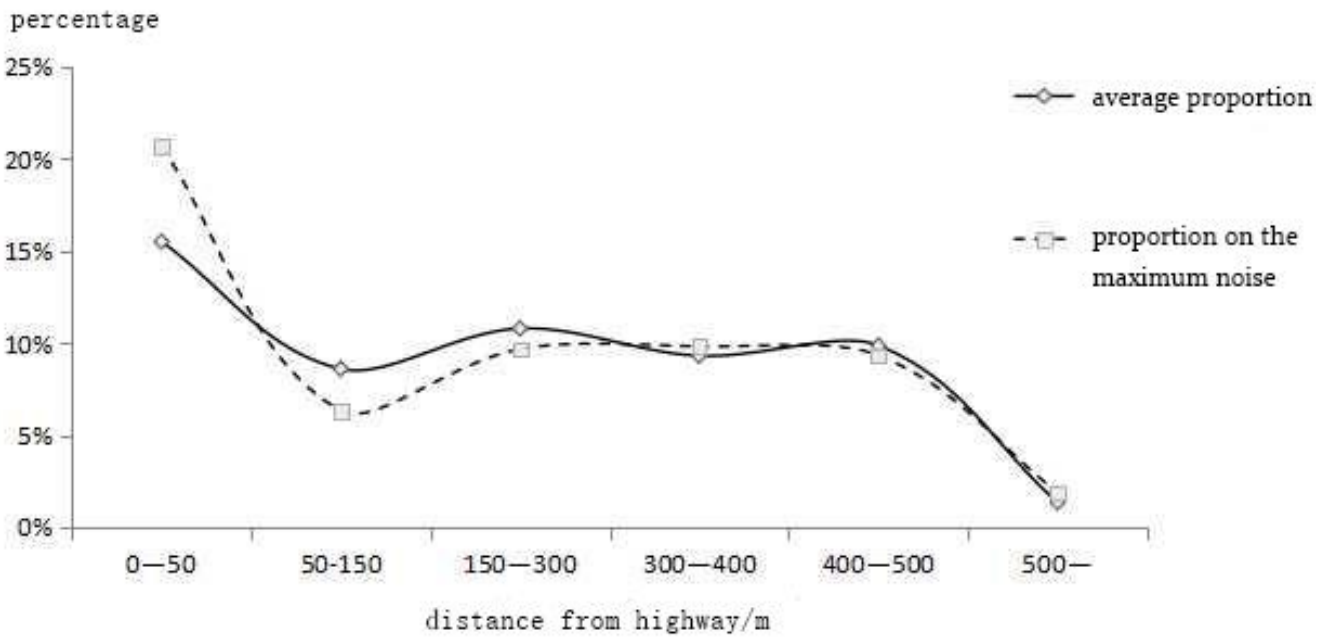

(c) Movement 


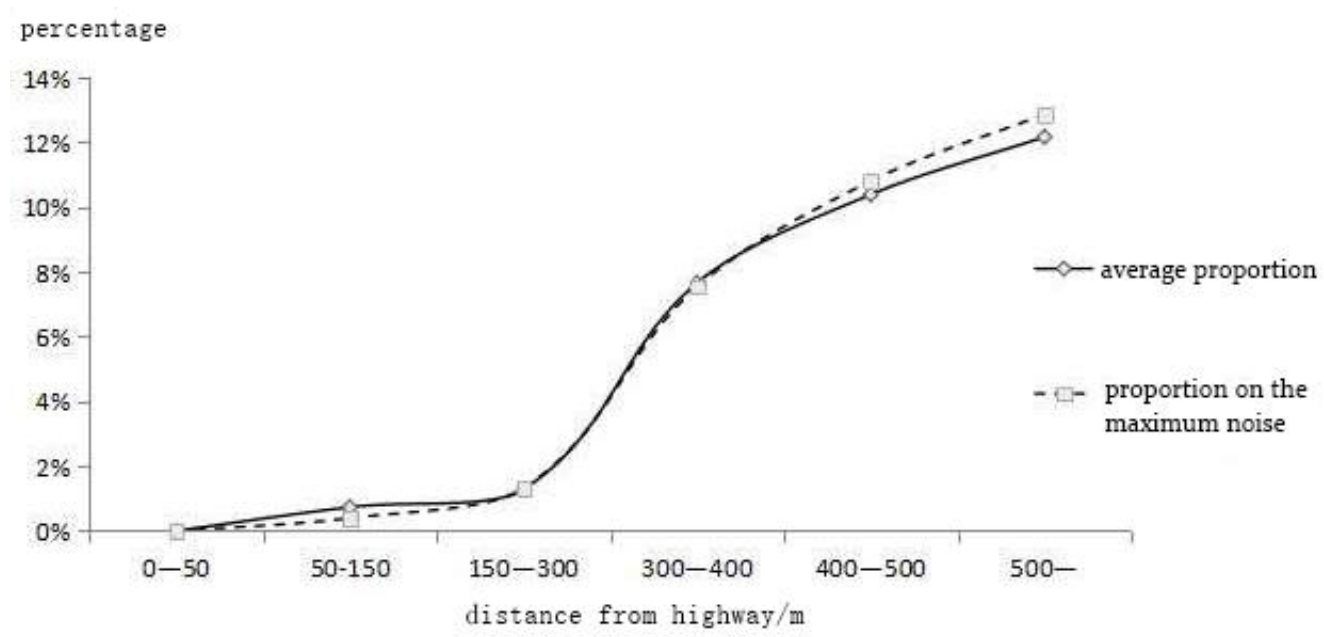

(d) Rest and others

Figure 5. Changes in Tibetan antelope behavior with different levels of traffic noise intensity

The proportions of the four main types of Tibetan antelope behavior changed with the distance and level of traffic noise intensity. The analysis showed similarities in how each kind of behavior was affected by the level of traffic noise intensity. The percentage of each behavior for the average noise and maximum noise at the same distance from the highway were slightly different, which indicates different impacts of different levels of traffic noise intensity on all types of Tibetan antelope behavior. However, the changes in the proportions of Tibetan antelope behaviors due to the average noise and maximum noise were not significant.

As shown in Figure 4(a), the proportion of foraging behavior before and after traffic noise showed a significant change $(4.19 \%) 0-50 \mathrm{~m}$ from the highway. The proportion showed a comparatively small change (1.06\%) 150-300 m from the highway. There were also changes in the proportion at $50-100 \mathrm{~m}$ and $300 \mathrm{~m}$ from the highway, but the changes were less than $1 \%$. Therefore, the noise-effect zone of the highway for the foraging behavior of Tibetan antelope was $0-50 \mathrm{~m}$ from the highway.

As shown in Figure $4(b)$, the most significant change $(3.69 \%)$ in the proportion of vigilance behavior before and after traffic noise was at $50-150 \mathrm{~m}$ from the highway. Changes at $0-50 \mathrm{~m}$ and $100 \mathrm{~m}$ from the highway did not exceed $1 \%$. Therefore, the noise-effect zone of the highway for the vigilance behavior of Tibetan antelope was 50$150 \mathrm{~m}$ from the highway.

As shown in Figure 4(c), the proportion of movement behavior changed significantly $(5.18 \%)$ before and after traffic noise at $0-50 \mathrm{~m}$ from the highway. There were relatively small changes of $2.27 \%$ and $1.12 \%$ at $0-50 \mathrm{~m}$ and $50-100 \mathrm{~m}$, respectively, from the highway. However, the change was less than $1 \%$ at $100 \mathrm{~m}$ from the highway. Therefore, the noise-effect zone of the highway for the movement behavior of Tibetan antelope was 0-300 $\mathrm{m}$ from the highway with a particularly strong effect within $50 \mathrm{~m}$.

As shown in Figure $4(d)$, there were no significant changes in the proportions of rest and other behaviors before and after traffic noise at different distances from the highway. Thus, the noise-effect zone of the highway for the rest and other behaviors of Tibetan antelope could not be determined.

In conclusion, the main significant changes in the proportions of four kinds of Tibetan antelope behavior before and after traffic noise changed within $150 \mathrm{~m}$ from the 
highway. In other words, the noise-effect zone of the highway on the behavior of Tibetan antelope was $0-150 \mathrm{~m}$ from the highway.

At $0-50 \mathrm{~m}$, the Tibetan antelope before and after traffic noise mainly exhibited decreased foraging behavior and increased movement behavior. In addition, the vigilance behavior fluctuated. When the vehicle was closer to the Tibetan antelope, some individuals stopped foraging and moved away from the highway, which caused the foraging behavior to decrease and movement behavior to increase. The vigilance behavior did not show a significant increase as expected but showed no irregular fluctuations. This is because the Tibetan antelope maintained a high degree of vigilance within $50 \mathrm{~m}$ from the highway with no radical reactions.

At $50-150 \mathrm{~m}$, the Tibetan antelope behavior before and after traffic noise mainly exhibited decreased movement behavior and increased vigilance behavior. When the vehicle was closer to the Tibetan antelope population, some individuals stopped moving and exhibited vigilance behavior.

\section{Discussion}

The impact of traffic noise on wildlife has been extensively studied in other countries, although not for high-altitude areas like the Qinghai-Tibet Plateaus. In this study, 500 datasets for the average and instantaneous noise levels at different distances along the roadside within $500 \mathrm{~m}$ from the highway were collected, and 1033 Tibetan antelopes were observed in a field experiment.

The behavior of wild ungulates in the area of $0-500 \mathrm{~m}$ had a significant difference from $2000 \mathrm{~m}-3000 \mathrm{~m}$ from the highway (Ying et al., 2007). And the avoidance distance of Tibetan antelope is $286.31 \mathrm{~m}$ from the highway (Lian et al., 2012). In this study, the results indicate that the noise-effect zone for the behavior of Tibetan antelope was 0$150 \mathrm{~m}$ from the highway. This suggests that new highways built in high-altitude areas should be at least $500 \mathrm{~m}$ from the activity area of Tibetan antelopes to protect them from the noise-effect zone. More careful consideration of the impact of traffic noise on wildlife is important when planning a new highway. Measures that can be taken in the design phase include changing the pavement material or decreasing the designed speed of vehicles. For Qinghai-Tibet Highway, sound insulation measures may help with avoiding the negative effects of traffic noise on the migration and reproduction of the Tibetan antelope population. For instance, sound barriers can be set on both side of the route along the animal channel, and natural shelter and traffic signs can be set up to prompt drivers to slow down and ban the horn.

The present study was preliminary in nature because it was a short-term study. The long-term impact of traffic noise on wildlife was not considered. More year-round research is needed to better understand the impact of traffic noise on Tibetan antelope and other wildlife. And further research can focus on the performance of sound monitoring at different heights and distances from the highway.

\section{Conclusion}

In our study, the noise intensity level showed a nonlinear negative correlation with the distance from the highway. Different levels of traffic noise intensity were found to have different impacts on all types of Tibetan antelope behaviors. Traffic levels were found to have the most significant effect on Tibetan antelope behavior within $150 \mathrm{~m}$ of 
the highway. The Tibetan antelope before and after impacted by traffic noise mainly exhibited decreased foraging behavior and increased movement behavior.

Acknowledgements. This research was supported in part by the National Key Research and Development Program of China (No. 2016YFC0802208) and by the Natural Science Foundation of Shaanxi Province (No. 2017JQ5122).

\section{REFERENCES}

[1] Alexander, S. M., Waters, N. M. (2000): The effects of highway transportation corridors on wildlife: A case study of Banff National Park. - Transportation Research Part C: Emerging Technologies 8: 307-320.

[2] Diaz-Varela, E. R., Vazquez-Gonzalez, I., Marey-Pérez, M. F. (2011): Assessing methods of mitigating wildlife-vehicle collisions by accident characterization and spatial analysis. - Transportation Research Part D: Transport and Environment 16: 281-287.

[3] Eigenbrod, F., Hecnar, S. J., Fahrig, L. (2009): Quantifying the road-effect zone: threshold effects of a motorway on anuran populations in Ontario, Canada. - Ecology and Society 14: 24 .

[4] Forman, R. T. T., Alexander, L. E. (1998): Roads and their major ecological effects. Annual Review of Ecology, Evolution and Systematics 29: 207-231.

[5] Gagnon, J. W., Schweinsburg, R. E., Dodd, N. L. (2007): Effects of Roadway Traffic on wild ungulates: a review of the literature and a case study of elk in Arizona. Proceedings of the International Conference on Ecology and Transportation 2001: 449458.

[6] Halfwerk, W., Holleman, L. J. M., Lessells, C. M., Slabbekoorn, H. (2011): Negative impact of traffic noise on avian reproductive success. - Journal of Applied Ecology 48: 210-219.

[7] Lian, X. M., Li, X. X., Xu, T. (2012): Avoidance distances of four ungulates from roads in Hoh-xil and related protection suggestions. - Chinese Journal of Ecology 31: 81-86.

[8] Xia, L., Yang, Q, Li, Z. (2007): The effect of the Qinghai-Tibet railway on the migration of Tibetan antelope Pantholops hodgsonii in Hoh-xil National Nature Reserve, China. Oryx 41: 352-357.

[9] Mcclure, C. J. W., Ware, H. E., Carlisle, J. (2013): An experimental investigation into the effects of traffic noise on distributions of birds: avoiding the phantom road. - Proceedings of the Royal Society B: Biological Sciences 280: 2290.

[10] Parris, K. M., Schneider, A. (2009): Impacts of Traffic Noise and Traffic Volume on Birds of Roadside Habitats. - Ecology and Society 14: 1698-1707.

[11] Samson, A., Ramakrishnan, B., Veeramani, A. (2016): Effect of vehicular traffic on wild animals in Sigur Plateau, Tamil Nadu, India. - Journal of Threatened Taxa 8: 9182-9189.

[12] Slabbekoorn, H., Ripmeester, E. A. P. (2008): Birdsong and anthropogenic noise: implications and applications for conservation. - Molecular Ecology 17: 72-83.

[13] Wood, W. E., Yezerinac, S. M. (2006): Song Sparrow (Melospiza melodia) song varies with urban noise. - The Auk 123: 650-659.

[14] Ying, B. F., Yu, Z. Y., Yang, S. M. (2007): Effect of Qinghai-Tibetan highway on the activities of Pantholops hodgsoni, Procapra picticaudata and Equus kiang. - Chinese Journal of Ecology 26: 810-816.

[15] Zande, A. N. V. D., Keurs, W. J. T., Weijden, W. J. V. D. (1980): The impact of roads on the densities of four bird species in an open field habitat - Evidence of a long-distance effect. - Biological Conservation 18: 299-321. 